\title{
EDITORIAL
}

\section{A LOOK ON THE BRIGHT SIDE}

\author{
A lot has been written recently about the woes of pharmaceutical companies in 2004 . With so \\ much bad press, it would be easy to overlook some of the encouraging developments last year \\ that might help the industry's recovery.
}

On the face of it, an annus horribilis seems a fair, if rather overused, description of 2004 for the pharmaceutical industry. The year began with the controversy over data indicating a possible link between antidepressant use and suicidality that ultimately resulted in the addition of a 'black box' warning to all antidepressant labels. And it closed with the withdrawal of the selective COX2 inhibitor rofecoxib (Vioxx) owing to cardiovascular side effects - a problem that is not only threatening the future of the whole drug class, but one that might also lead to changes in drug regulation that could make drug development even more risky and expensive for companies.

In part owing to the general perception of the role of the pharmaceutical industry and its regulators in these two episodes, 2004 might well be the nadir of public confidence in the industry as a whole. Widely publicized books attacking the high prices of branded drugs and lamenting the lack of innovation have further contributed to the dismal tone of most end-of-year stories on the industry.

But taking inspiration from a quote from the author Ralph Waldo Emerson - "When it is dark enough, you can see the stars" - were there any bright spots in the gloom of 2004? Well, as highlighted in the news story on page 93 , one of the most widely used benchmarks of the state of the industry - the annual number of New Molecular Entities (NMEs) approved by the FDA continued to show improvement. With 31 NMEs having been approved, last year was actually the most successful since 1999 when assessed in these simple terms. Furthermore, a number of these agents could be argued to be highly innovative, such as the anticancer antibody bevacizumab, the first drug to target tumour angiogenesis, whose approval finally validated a therapeutic hypothesis that was proposed more than 30 years ago. And a second anti-angiogenic agent, pegaptanib, which was approved for the treatment of age-related macular degeneration late last year, is the first to be based on a type of therapeutic RNA known as an aptamer.
Also related to RNA, although much earlier in the pipeline, there was encouraging progress in the therapeutic application of RNA interference (RNAi). This technology has been widely and rapidly adopted in target validation in recent years owing to its power to specifically and efficiently 'silence' genes. But a number of key issues, most notably associated with delivery, still pose similar challenges for the clinical use of RNAi as have plagued other nucleic-acid-based strategies, such as antisense. However, research published last year describing a method to effectively deliver RNAi therapy systemically ${ }^{1}$ suggests that these challenges could at last be surmountable. The first clinical trials of RNAi were also announced, which is remarkable in itself given that the technique came on to the scene little more than five years ago.

There have also been positive developments from regulatory agencies. Last year, the FDA released its 'Critical Path' white paper', which aims to bring attention and focus to the need for targeted efforts to improve the tools and techniques used to evaluate the safety, efficacy and quality of drug products. Mentioned in this report is the future development of pharmacogenetics, and the latter part of 2004 saw the approval in the United States and Europe of the first microarraybased pharmacogenetic test, which allows drug doses to be optimized on the basis of individual variations in drug-metabolizing enzymes. A growth in the application of pharmacogenetic testing will mark the longpredicted movement from the traditional blockbuster model of drug discovery and development towards drugs that work better in selected populations, and could also be valuable in helping to avoid further drug safety problems such as those that have rocked the industry in the past year.

1. Soutschek, J. et al. Therapeutic silencing of an endogenous gene by systemic administration of modified siRNAs. Nature 432, 173-178 (2004)

2. Innovation or Stagnation? Challenge and Opportunity on the Critical Path to New Medical Products [online], <http://www.fda.gov/oc/initiatives/ criticalpath/whitepaper.pdf> (2004) 